\title{
Optimal control problems on time scales described by Volterra integral equations on time scales
}

\author{
Problemas de controle ótimo em escalas temporais descritos por \\ equações integrais de Volterra em escalas temporais
}

\author{
Iguer Santos
}

iguerluis@mat.feis.unesp.br

\begin{abstract}
In this work we consider two class of optimal control problems on time scales described by Volterra integral equations on time scales. We have established the existence of solutions for these two class of optimal control problems.

Keywords: Optimal control, Volterra integral equations, time scales.
\end{abstract}

\section{Resumo}

Neste trabalho nós consideramos duas classes de problemas de controle ótimo em escalas temporais descritos por equações integrais de Volterra em escalas temporais. Nós estabelecemos a existência de soluções para essas duas classes de problemas de controle ótimo.

Palavras-chave: Controle ótimo, equações integrais de Volterra, escalas temporais. 


\section{Introduction}

Results of existence of optimal controls for optimal control problems on time scales are to be found in [Santos e Silva (2014), Theorem 5.2], [Liu et al. (2011), Theorem 4.3], [Peng et al. (2011), Theorem 4.3], [Zhan et al. (2012), Theorem 4.1] and [Zhan e Wei (2009), Theorem 4.2].

Using the theorems [dos Santos (2015), Theorem 3.3] and [dos Santos (2015), Theorem 3.4], we give sufficient conditions for the existence of optimal controls to optimal control problems on time scales described by Volterra integral equations on time scales. To the best of our knowledge, the optimal control problems of a system governed by the Volterra integral equations on time scales have not been considered in the literature.

\section{Preliminaries}

A time scale $\mathbb{T}$ is a nonempty closed subset of real numbers. Here we use an arbitrary bounded time scale $\mathbb{T}$ such that $a=\min \mathbb{T}<$ $b=\max \mathbb{T}$.

Define the forward jump operator $\sigma: \mathbb{T} \rightarrow$ $\mathbb{T}$ by

$$
\sigma(t)=\inf \{s \in \mathbb{T}: s>t\}
$$

where inf $\varnothing=\sup \mathbb{T}$.

Let $c, d \in \mathbb{T}$ be such that $c \leq d$. Given a function $f: \mathbb{T} \rightarrow \mathbb{R}$, we indicate the Riemann $\Delta$-integral of $f$ on $[c, d]_{\mathbb{T}}$ by

$$
\int_{c}^{d} f(s) \Delta s
$$

For functions $f: \mathbb{T} \rightarrow \mathbb{R}^{n}$ the integration is considered componentwise.

We study optimal control problems on time scales described by the following Volterra integral equations on time scales

$$
x(t)=u(t)+\int_{a}^{t} g(t, s, x(s)) \Delta s
$$

$$
x(t)=u(t)+\int_{a}^{t} g(t, s, x(\sigma(s))) \Delta s
$$

where $x: \mathbb{T} \rightarrow \mathbb{R}^{n}$ is the unknown function, $g: \mathbb{T} \times \mathbb{T} \times \mathbb{R}^{n} \rightarrow \mathbb{R}^{n}$ and $f: \mathbb{T} \rightarrow \mathbb{R}^{n}$ are given functions, and $t \in \mathbb{T}$. The existence of continuous solutions to Eqs. (1) and (2) can be found in Kulik e Tisdell (2008).

Below we state the convergence results of solutions used to establish the existence of optimal controls to optimal control problems on time scales described by Volterra integral equations on time scales.

Theorem 2.1 (dos Santos (2015)). Let $\left\{g_{k}\right\}$ be a sequence of continuous functions with $g_{k}: \mathbb{T} \times$ $\mathbb{T} \times \mathbb{R}^{n} \rightarrow \mathbb{R}^{n}$ satisfying

$$
\left\|g_{k}(t, s, x)\right\| \leq C(1+\|x\|)
$$

on its domain. Suppose that $\left\{f_{k}\right\}$ is a sequence of uniformly bounded and equicontinuous functions with $f_{k}: \mathbb{T} \rightarrow \mathbb{R}^{n}$ and $\left\|f_{k}-f\right\|_{\infty} \rightarrow 0$. Suppose also

1. $g_{k}(t, s, x) \rightarrow g(t, s, x)$ on $\mathbb{T} \times \mathbb{T} \times \mathbb{R}^{n}$;

2. there exists $L>0$ such that $\| g_{k}(t, s, x)-$ $g_{k}(t, s, y)\|\leq L\| x-y \|$ for each $k$;

3. for each $k, \psi_{k}(t)$ is a solution of

$$
\psi_{k}(t)=f_{k}(t)+\int_{a}^{t} g_{k}\left(t, s, \psi_{k}(s)\right) \Delta s,
$$

$t \in \mathbb{T}$

4. given $\epsilon>0$ and $M>0$, there exists $\delta>0$ such that

$$
\left\|g_{k}(t, s, x)-g_{k}\left(t_{1}, s, x\right)\right\| \leq \epsilon\left|t-t_{1}\right|
$$

when $s \in \mathbb{T},\left|t-t_{1}\right|<\delta, t, t_{1} \in \mathbb{T},\|x\| \leq$ $M$ and $k$ is an integer.

Then there is a subsequence $\left\{\psi_{k_{j}}\right\} \subset\left\{\psi_{k}\right\}$ and $a$ function $\psi: \mathbb{T} \rightarrow \mathbb{R}^{n}$ such that $\left\|\psi_{k_{j}}-\psi\right\|_{\infty} \rightarrow 0$, and $\psi$ satisfies

$$
\psi(t)=f(t)+\int_{a}^{t} g(t, s, \psi(s)) \Delta s
$$

on $\mathbb{T}$. 
Theorem 2.2 (dos Santos (2015)). Take a sequence of continuous functions $g_{k}: \mathbb{T} \times \mathbb{T} \times \mathbb{R}^{n} \rightarrow \mathbb{R}^{n}$ such that

$$
\left\|g_{k}(t, s, x)\right\| \leq C(1+\|x\|)
$$

for all $(t, s, x) \in \mathbb{T} \times \mathbb{T} \times \mathbb{R}^{n}$. Consider a sequence of uniformly bounded and equicontinuous functions $f_{k}: \mathbb{T} \rightarrow \mathbb{R}^{n}$ with $\left\|f_{k}-f\right\|_{\infty} \rightarrow 0$. Consider also the hypotheses

1. $g_{k}(t, s, x) \rightarrow g(t, s, x)$ on $\mathbb{T} \times \mathbb{T} \times \mathbb{R}^{n}$;

2. there exists $L>0$ such that $\| g_{k}(t, s, x)-$ $g_{k}(t, s, y)\|\leq L\| x-y \|$ for each $k ;$

3. for each $k, \psi_{k}(t)$ is a solution of

$$
\begin{aligned}
& \psi_{k}(t)=f_{k}(t)+\int_{a}^{t} g_{k}\left(t, s, \psi_{k}(\sigma(s))\right) \Delta s, \\
& t \in \mathbb{T} ;
\end{aligned}
$$

4. for each $\epsilon>0$ and $M>0$, there exists $\delta>0$ such that

$$
\left\|g_{k}(t, s, x)-g_{k}\left(t_{1}, s, x\right)\right\| \leq \epsilon\left|t-t_{1}\right|
$$

whenever $s \in \mathbb{T},\left|t-t_{1}\right|<\delta, t, t_{1} \in \mathbb{T}$, $\|x\| \leq M$ and $k$ is an integer.

If $C(b-a)<1$, then there is a subsequence $\left\{\psi_{k_{j}}\right\} \subset$ $\left\{\psi_{k}\right\}$ and a function $\psi: \mathbb{T} \rightarrow \mathbb{R}^{n}$ such that $\left\|\psi_{k_{j}}-\psi\right\|_{\infty} \rightarrow 0$, and $\psi$ satisfies

$$
\psi(t)=f(t)+\int_{a}^{t} g(t, s, \psi(\sigma(s))) \Delta s
$$

on $\mathbb{T}$.

\section{Optimal control problems on time scales}

We denote the set of all continuous functions with domain $\mathbb{T}$ and taking values on $\mathbb{R}^{n}$ by $C\left(\mathbb{T}, \mathbb{R}^{n}\right)$. Let $\mathcal{U} \subset \mathcal{K}$, where $\mathcal{K}$ is the set of all functions $f: \mathbb{T} \rightarrow \mathbb{R}^{n}$ satisfying

$$
\|f(t)-f(s)\| \leq K|t-s|, \forall t, s \in \mathbb{T}
$$

for some constant $K>0$.

Consider the following optimal control problem $\left(P_{1}\right)$ :

$$
\min h(x(a), x(b))
$$

on all pairs $(x, u) \in C\left(\mathbb{T}, \mathbb{R}^{n}\right) \times \mathcal{U}$, such that

$$
\left\{\begin{array}{l}
x(t)=u(t)+\int_{a}^{t} g(t, s, x(s)) \Delta s, t \in \mathbb{T} \\
(x(a), x(b)) \in E
\end{array}\right.
$$

where $E \subset \mathbb{R}^{2 n}$ is a closed set and $h: \mathbb{R}^{n} \times$ $\mathbb{R}^{n} \rightarrow \mathbb{R}$ is a lower semicontinuous function.

We say that $(x, u) \in C\left(\mathbb{T}, \mathbb{R}^{n}\right) \times \mathcal{U}$ is an admissible process for $\left(P_{1}\right)$, if the pair $(x, u)$ satisfies the Eq. (1) and $x$ obeys the condition $(x(a), x(b)) \in E$. A process $(\bar{x}, \bar{u})$ is called an optimal process for $\left(P_{1}\right)$, if it is an admissible process for $\left(P_{1}\right)$ that satisfies

$$
h(\bar{x}(a), \bar{x}(b)) \leq h(x(a), x(b))
$$

for all admissible process $(x, u)$ of $\left(P_{1}\right)$.

Theorem 3.1. Let $g: \mathbb{T} \times \mathbb{T} \times \mathbb{R}^{n} \rightarrow \mathbb{R}^{n}$ be $a$ continuous function satisfying

$$
\|g(t, s, x)\| \leq C(1+\|x\|)
$$

on its domain. Suppose that

1. there exists $L>0$ such that $\| g(t, s, x)-$ $g(t, s, y)\|\leq L\| x-y \|$

2. given $\epsilon>0$ and $M>0$, there exists $\delta>0$ such that

$$
\left\|g(t, s, x)-g\left(t_{1}, s, x\right)\right\| \leq \epsilon\left|t-t_{1}\right|
$$

when $s \in \mathbb{T},\left|t-t_{1}\right|<\delta, t, t_{1} \in \mathbb{T}$ and $\|x\| \leq M$.

Then $\left(P_{1}\right)$ has an optimal process.

Proof. Denote by $\inf \left\{P_{1}\right\}$ the greatest lower bound on $h(x(a), x(b))$ over admissible processes $(x, u)$ of $\left(P_{1}\right)$. Hence there exists a sequence of admissible processes $\left(x_{i}, u_{i}\right)$ of $\left(P_{1}\right)$ obeying

$$
\inf \left\{P_{1}\right\}=\lim h\left(x_{i}(a), x_{i}(b)\right) .
$$


Since $\left\{u_{i}\right\}$ is a equicontinuous sequence, from Arzela-Ascoli's Theorem there exists a subsequence of $\left\{u_{i}\right\}$, we do not relabel, such that $\left\|u_{i}-\bar{u}\right\|_{\infty} \rightarrow 0$. It follows from [dos Santos (2015), Theorem 3.3] that there is a subsequence of $\left\{x_{i}\right\}$, we do not relabel, such that $\left\|x_{i}-\bar{x}\right\|_{\infty} \rightarrow 0$. Moreover, the function $\bar{x}:$ $\mathbb{T} \rightarrow \mathbb{R}^{n}$ satisfies

$$
\bar{x}(t)=\bar{u}(t)+\int_{a}^{t} g(t, s, \bar{x}(s)) \Delta s
$$

on $\mathbb{T}$.

As $\left(x_{i}(a), x_{i}(b)\right) \in E$ and $E$ is a closed set, we conclude that $(\bar{x}(a), \bar{x}(b)) \in E$ and then $(\bar{x}, \bar{u})$ is an admissible process for $\left(P_{1}\right)$. We also have

$$
\begin{aligned}
\inf \left\{P_{1}\right\} & =\lim h\left(x_{i}(a), x_{i}(b)\right) \\
& =\liminf h\left(x_{i}(a), x_{i}(b)\right) \\
& \geq h(\bar{x}(a), \bar{x}(b)) \geq \inf \left\{P_{1}\right\}
\end{aligned}
$$

and thence $(\bar{x}, \bar{u})$ is an optimal process for $\left(P_{1}\right)$.

Now consider the following optimal control problem $\left(P_{2}\right)$ :

$$
\min h(x(a), x(b))
$$

on all pairs $(x, u) \in C\left(\mathbb{T}, \mathbb{R}^{n}\right) \times \mathcal{U}$, such that

$$
\left\{\begin{array}{l}
x(t)=u(t)+\int_{a}^{t} g(t, s, x(\sigma(s))) \Delta s, t \in \mathbb{T} \\
(x(a), x(b)) \in E .
\end{array}\right.
$$
$\left(P_{2}\right)$.

Below we get the existence of solutions to

Theorem 3.2. Assume that $g: \mathbb{T} \times \mathbb{T} \times \mathbb{R}^{n} \rightarrow$ $\mathbb{R}^{n}$ is a continuous function satisfying

$$
\|g(t, s, x)\| \leq C(1+\|x\|)
$$

on its domain. We also suppose that

1. there exists $L>0$ such that $\| g(t, s, x)-$ $g(t, s, y)\|\leq L\| x-y \| ;$
2. for each $\epsilon>0$ and $M>0$, there exists $\delta>0$ such that

$$
\left\|g(t, s, x)-g\left(t_{1}, s, x\right)\right\| \leq \epsilon\left|t-t_{1}\right|
$$

whenever $s \in \mathbb{T},\left|t-t_{1}\right|<\delta, t, t_{1} \in \mathbb{T}$ and $\|x\| \leq M$.

If $C(b-a)<1$, then $\left(P_{2}\right)$ has an optimal process.

Proof. Let $\inf \left\{P_{2}\right\}$ denote the greatest lower bound on $h(x(a), x(b))$ over admissible processes $(x, u)$ of $\left(P_{2}\right)$. Thence there exists a sequence of admissible processes $\left(x_{i}, u_{i}\right)$ of $\left(P_{2}\right)$ obeying

$$
\inf \left\{P_{2}\right\}=\lim h\left(x_{i}(a), x_{i}(b)\right) .
$$

As $\left\{u_{i}\right\}$ is a equicontinuous sequence, it follows from Arzela-Ascoli's Theorem that there exists a subsequence of $\left\{u_{i}\right\}$, we do not relabel, such that $\left\|u_{i}-\bar{u}\right\|_{\infty} \rightarrow 0$. From [dos Santos (2015), Theorem 3.4] there exists a subsequence of $\left\{x_{i}\right\}$, we do not relabel, such that $\left\|x_{i}-\bar{x}\right\|_{\infty} \rightarrow 0$. Furthermore, the pair $(\bar{x}, \bar{u})$ satisfies the equation

$$
\bar{x}(t)=\bar{u}(t)+\int_{a}^{t} g(t, s, \bar{x}(\sigma(s))) \Delta s
$$

on $\mathbb{T}$.

Since $\left(x_{i}(a), x_{i}(b)\right) \in E$ and $E$ is a closed set, we have

$$
\lim \left(x_{i}(a), x_{i}(b)\right)=(\bar{x}(a), \bar{x}(b)) \in E
$$

and then $(\bar{x}, \bar{u})$ is an admissible process for $\left(P_{2}\right)$.

Finally, since

$$
\begin{aligned}
\inf \left\{P_{2}\right\} & =\lim h\left(x_{i}(a), x_{i}(b)\right) \\
& =\liminf h\left(x_{i}(a), x_{i}(b)\right) \\
& \geq h(\bar{x}(a), \bar{x}(b)) \geq \inf \left\{P_{2}\right\}
\end{aligned}
$$

we conclude that $(\bar{x}, \bar{u})$ is an optimal process for $\left(P_{2}\right)$. 


\section{References}

Kulik, T., Tisdell, C. C. (2008). Volterra integral equations on time scales: basic qualitative and quantitative results with applications to initial value problems on unbounded domains. Int J Difference Equ, 3(1), 103-133.

Liu, G., Xiang, X., Peng, Y. (2011). Nonlinear integro-differential equations and optimal control problems on time scales. Comput Math Appl, 61(2), 155-169.

Peng, Y., Xiang, X., Jiang, Y. (2011). Nonlinear dynamic systems and optimal control problems on time scales. ESAIM Control Optim Calc Var, 17(3), 654-681.

dos Santos, I. L. D. (2015). On volterra integral equations on time scales. Mediterr J Math, 12(2), 471-480.

Santos, I. L. D., Silva, G. N. (2014). Filippov's selection theorem and the existence of solutions for optimal control problems in time scales. Comput Appl Math, 33(1), 223-241.

Zhan, Z., Wei, W. (2009). On existence of optimal control governed by a class of the firstorder linear dynamic systems on time scales. Appl Math Comput, 215(6), 2070-2081.

Zhan, Z., Wei, W., Li, Y., Xu, H. (2012). Existence for calculus of variations and optimal control problems on time scales. Int J Innov Comput Inf Control, 8(5(B)), 3793-3808. 